\title{
Respiración disfuncional: una nueva vista a un mal longevo
}

\section{Dysfunctional breathing: a new look to a long-lived ailment}

\author{
Juan Gerardo Alcaraz-López, ${ }^{*}$ Oscar Joseph Camacho-Alamo*
}

*Instituto Mexicano del Seguro Social. Universidad de Guadalajara. México.

\begin{abstract}
RESUMEN. La respiración disfuncional es un trastorno heterogéneo caracterizado por diversas alteraciones en las variables que comprenden la mecánica y dinámica respiratoria. Agrupa diferentes tipos de patrones respiratorios anormales. Dentro del cual encontramos al síndrome de hiperventilación, respiración torácica dominante, suspiración periódica profunda, asincronía toracoabdominal y espiración forzada abdominal. Se ha identificado como un trastorno frecuente de manera aislada y como comorbilidad en pacientes con enfermedades respiratorias y cardíacas principalmente. Es escasamente conocida como entidad, sin embargo, su estudio es fundamental debido a que la evidencia muestra una carga significativa sobre la calidad de vida. El reconocimiento es el elemento más fuerte del abordaje en estos pacientes y, aunque no cuenta con un abordaje diagnóstico claramente definido, en los últimos años han surgido pruebas que parecen prometedoras para la evaluación del patrón respiratorio. Los tratamientos actuales muestran tendencia positiva en cuanto a diversos desenlaces. Se tiene una oportunidad futura para desarrollar ensayos clínicos que permitan detallar estas terapias y dirigirlas hacia los diversos subtipos de la respiración disfuncional. Con la presente revisión se pretende brindar un panorama claro, explicando de manera general los elementos que intervienen en la fisiopatología, además de presentar una panorámica sobre las pruebas diagnósticas y tratamientos. Esperamos aportar una perspectiva distinta del trastorno que permita el diseño de ensayos clínicos venideros.
\end{abstract}

Palabras clave: Respiración disfuncional, síndrome de hiperventilación, entrenamiento respiratorio, patrones respiratorios.

\section{INTRODUCCIÓN}

La respiración disfuncional es un trastorno heterogéneo caracterizado por diversas alteraciones en las variables que comprenden la mecánica y dinámica respiratoria. Agrupa diferentes tipos de patrones respiratorios anormales (Figura 1).,2 Dentro de éstos encontramos al síndrome de hiperventilación,

\section{Correspondencia:}

\section{Dr. Juan Gerardo Alcaraz-López}

Instituto Mexicano del Seguro Social. Universidad de Guadalajara. Correo electrónico: alcarazjuang@gmail.com

Recibido: 01-II-2021; aceptado: 23-IV-2021.

Citar como: Alcaraz-López JG, Camacho-Alamo OJ. Respiración disfuncional: una nueva vista a un mal longevo. Neumol Cir Torax. 2021; 80 (3): 188-196. https://dx.doi.org/10.35366/102479
ABSTRACT. Dysfunctional breathing is a term that groups together different types of abnormal breathing patterns. It has been identified as a common disorder; in isolation and as a comorbidity in patients with respiratory and cardiac diseases specially. It is poorly known as an entity; however, its study is essential because the body of evidence show a significant burden on quality of life. Recognition is the strongest element of the approach in these patients and, although it does not have a clearly defined diagnostic approach, in recent years, tests have emerged that seem promising for the evaluation of the respiratory pattern. Current treatments show a positive trend in various outcomes. There is a future opportunity to develop clinical trials to detail these therapies and target the various subtypes of dysfunctional breathing. The present review aims to provide a clear picture, explaining in a general manner the elements that intervene in pathophysiology, in addition to presenting a perspective on diagnostic tests and treatments. We hope to provide a different perspective of the disorder that will allow the design of future clinical trials.

Keywords: Dysfunctional breathing, hyperventilation syndrome, respiratory breathing, breathing patterns.

respiración torácica dominante, suspiración periódica profunda, asincronía toracoabdominal y espiración forzada abdominal. ${ }^{2}$ Puede ocurrir de manera primaria (i.e., debido a causas psicogénicas como la ansiedad y trastorno de pánico) $)^{3,4}$ o secundaria a enfermedad cardíaca, pulmonar o neurológica (ej. asma). ${ }^{5,6}$

El estudio de la respiración disfuncional inició con la identificación del subtipo ahora más reconocido, el síndrome de hiperventilación. Desde hace un decalustro éste se empezó a estudiar, ${ }^{7}$ uno de los primeros autores en brindar una definición formal fue Brashear en 1983. ${ }^{8}$ Posteriormente, en el artículo pionero de Gilbert C. ${ }^{4}$ se hace mención por primera vez a la respiración disfuncional.

$$
\text { Epidemiología }
$$

Es ampliamente desconocida debido a que no hay criterios diagnósticos claramente definidos. Sin embargo, usando 
los métodos de diagnóstico actuales, principalmente el cuestionario de Nijmegen, la respiración disfuncional se ha reportado que ocurre en $10 \%$ de la población general (siendo más frecuente en mujeres), $5,9,10 \sim 70 \%$ en pacientes con trastorno de ansiedad, ${ }^{11}$ en $27 \%$ en pacientes con asma (siendo más frecuente en pacientes con asma severo) y de $47 \%$ en pacientes con EPOC (más usual en EPOC severo). ${ }^{10}$ Aunque es notable que estos estudios no diferencian entre los diferentes tipos de respiración disfuncional.

\section{Fisiología}

La respiración requiere de un programa sofisticado para ventilar los pulmones y responder adecuadamente a los retos fisiológicos y a las condiciones externas cambiantes.

La respiración se origina de un patrón generador central en el tallo cerebral, que consiste en una red de microcircuitos que generan ritmos y patrones. ${ }^{12}$ Se ha visto que varios de estos centros son influenciados por las emociones y la cognición (Figura 2). ${ }^{13}$

La respiración se divide en tres fases: la inspiración, la posinspiración y la espiración. La primera fase tiene como objetivo la movilización de gas desde la atmósfera a través de un sistema de baja presión constituido por los pulmones y la caja torácica, lo que permite que el aire alcance los capilares pulmonares y consiguientemente se logre la hematosis, esto gracias a la expansión efectiva de la caja torácica dada por la contracción del diafragma y los múscu- los intercostales externos que genera una presión negativa entre la pleura y los pulmones, lo que permite que éstos se expandan. ${ }^{14}$

La posinspiración consta de una modulación de la respiración durante «conductas no ventilatorias», tales como la deglución y vocalización, entre otras. Esto mediante un aumento de la resistencia de la vía aérea superior debido a una contracción de los músculos aductores laríngeos, lo que permite contrarrestar el retroceso del pulmón expandido y detener el flujo de aire espirado. ${ }^{15}$

La última fase es la espiración, ésta contempla la disminución de la presión intrapleural e intratorácica, la cual permite que la presión intersticial y alveolar sean neutras o levemente positivas con respecto a la presión atmosférica, facilitado por la acción de los músculos intercostales internos y el diafragma, estos cambios de presión permiten el flujo de gas inspiratorio y espiratorio. ${ }^{14,16}$

La posinspiración beneficia al intercambio gaseoso alveolar y disminuye la posibilidad de colapso alveolar. ${ }^{16,17}$ La modulación de la resistencia de la vía aérea durante la posinspiración también contribuye a los reflejos expulsivos como la $\operatorname{tos}^{17-19}$ y a los comportamientos no respiratorios, como la deglución ${ }^{18,20-23}$ o la vocalización. ${ }^{24}$

La eupnea se define como una frecuencia respiratoria en reposo de entre 12-16 respiraciones por minuto, ésta requiere de una inspiración activa, con una posinspiración y espiración, en su mayoría pasivas. ${ }^{12}$ La espiración se hace activa durante el ejercicio vigoroso cuando la demanda metabólica incrementa, en altitudes donde los niveles de
Síndrome de hiperventilación Es el subtipo más estudiado y reconocido. Debe cumplir con criterios como hipocapnia y alcalosis respiratoria en el contexto de hiperventilación
Suspiración periódica profunda Suspiros frecuentes relacionados o no a patología de base, suele acompañarse de hiperventilación
Respiración torácica dominante También llamada apical, suele presentarse en pacientes con incremento en las necesidades ventilatorias, existiendo falta de expansión costal lateral con predominancia en tórax superior

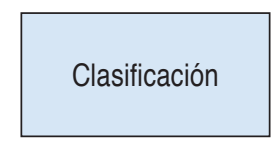

Espiración forzada abdominal Asociado a pacientes con EPOC, los pacientes presentan contracción excesiva del abdomen para ayudar a la espiración
A sincronía toracoabdominal

Presente en pacientes con obstrucción de vías respiratorias altas o trastornos neuromusculares, existe un retraso entre el movimiento del tórax y el abdomen, dando como resultado respiración disfuncional

Figura 1: Clasificación de la respiración disfuncional. Tomado de: Boulding $\mathrm{R}$ et al. ${ }^{2}$ 


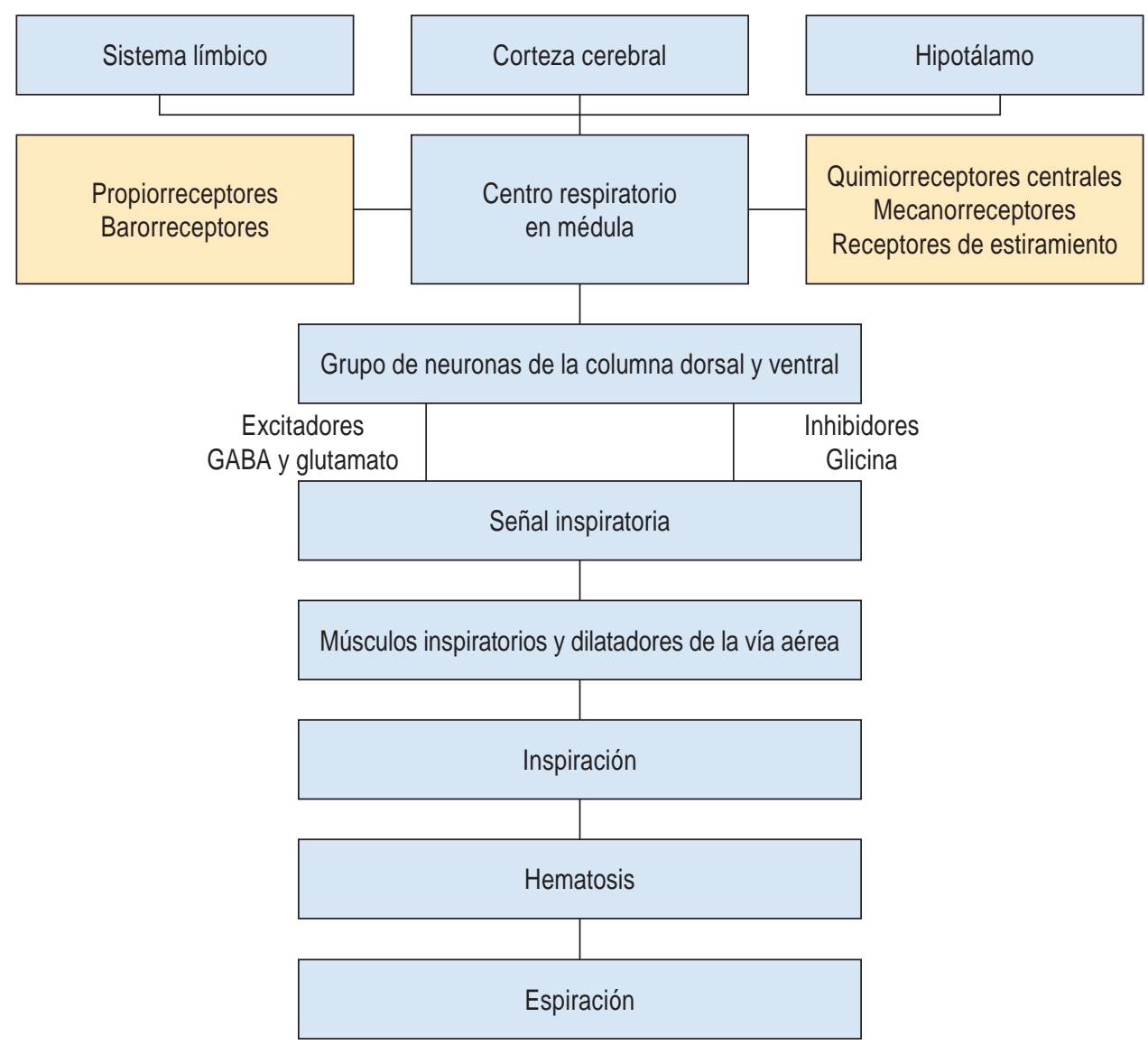

Figura 2:

Fisiología de la respiración. Modificado de: Gómez CMH. ${ }^{14}$

GABA = ácido gamma aminobutírico. oxígeno se reducen y de manera intermitente con el sueño durante la fase de movimientos oculares rápidos. ${ }^{12}$

La respiración es programada periódicamente (cada cinco minutos) por suspiros, éstos son esenciales para mantener una función pulmonar adecuada. ${ }^{12}$ La frecuencia de los suspiros es especialmente alta en infantes y durante períodos de hipoxia. Además, éstos también se generan asociados con emociones como alivio, aflicción, exasperación, agotamiento, anhelo, felicidad, entre otros. ${ }^{12}$

\section{Capacidad de adaptación del sistema respiratorio}

El patrón de la respiración es influenciado por las emociones, el estado del sistema nervioso autónomo y el estado mental. ${ }^{13}$ De manera que las respuestas de lucha o huida se correlacionan con respiraciones rápidas, por el contrario, la calma mental se asocia con respiración lenta, y los niveles bajos de estrés se asocian con respiración lenta y profunda. ${ }^{13}$

Adicionalmente, el patrón de la respiración se modifica ante condiciones como el crecimiento, la maduración, el embarazo, la edad, la enfermedad y la lesión (Figura 3). ${ }^{12}$

Los músculos accesorios de la respiración proveen un control postural y facilitan la respiración durante situaciones estresantes, el ejercicio, situaciones adversas en las cuales el flujo de aire está limitado, con lo cual el patrón respiratorio se adapta a las propiedades de la pared torácica, pulmón y vía aérea. ${ }^{25}$

\section{Manifestaciones clínicas}

Incluye síntomas respiratorios y no respiratorios. Entre los respiratorios el principal es la disnea, aunque también se pueden presentar con respiración dificultosa o incómoda. ${ }^{26}$ Entre los no respiratorios se encuentran palpitaciones, mareo, aturdimiento y distensión abdominal, que de hecho este último es parte del cuestionario usado para el tamizaje de este trastorno: el cuestionario de Nijmegen. ${ }^{27}$

De manera más específica, los síntomas relacionados con el síndrome de hiperventilación (el más reconocido) se asocian a la hipocapnia y a la alcalosis respiratoria, como lo son las parestesias, tetania y entumecimiento. ${ }^{26}$

Es notable que en ocasiones, debido al desconocimiento del síndrome, la presentación clínica puede confundirse con asma, para lo cual se requiere dirigir el abordaje diagnóstico de acuerdo con las características del paciente.

Otros síntomas que incluye este trastorno son relacionados a la psicopatología del individuo (los cuales varían ampliamente), ${ }^{7}$ que de manera directa o indirecta 
pueden afectar al patrón respiratorio como síntomas relacionados al trastorno de pánico y ansiedad generalizada, entre otros.

\section{Clasificación}

Los tipos de respiración disfuncional descritos en la literatura se detallan en la Figura 3.

\section{DIAGNÓSTICO}

El abordaje diagnóstico contempla identificar la enfermedad orgánica que pudo haber originado el trastorno, así como la indagación acerca de factores contribuyentes y perpetuantes del trastorno.

Un abordaje propuesto por Vidotto et al. ${ }^{28}$ toma en cuenta: aspectos biomecánicos (movimientos torácicos), síntomas respiratorios, aspectos fisiológicos (efectos cardiovasculares), aspectos sociales (calidad de vida), aspectos psicológicos (ansiedad y depresión, entre otros), aspectos bioquímicos ( $\mathrm{CO}_{2}$ al final de la espiración).

A continuación se describen algunos métodos diagnósticos para el síndrome de hiperventilación:

El cuestionario de Nijmegen es ampliamente usado en ensayos clínicos. ${ }^{29-31}$ Esta herramienta fue desarrollada y validada para el tamizaje del síndrome de hiperventilación, ${ }^{27}$ aunque en la actualidad, probablemente de manera inapropiada, se usa para el tamizaje de respiración disfuncional sin distinción de algún subtipo; sin embargo, en 32 años no se ha propuesto otra herramienta adecuada.

La prueba de provocación de la hiperventilación consiste en pedir al paciente que respire profunda y rápidamente por un determinado tiempo (típicamente 2 a 3 minutos) y posteriormente observar si se reproducen los síntomas con la prueba, si ese es el caso se diagnostica a la persona con respiración disfuncional. ${ }^{32}$ Esta prueba no diferencia entre los síntomas causados por la hipocapnia y aquellos causados por la ansiedad asociada con las indicaciones de la prueba o un malestar mecánico, por lo cual sobreestima el diagnóstico del síndrome de hiperventilación. ${ }^{33}$

Otra investigación utilizó la capnografía asociando los valores del $\mathrm{CO}_{2}$ al final de la espiración con la severidad de la hiperventilación: $<35 \mathrm{mmHg}$ indican hiperventilación leve, $<32 \mathrm{mmHg}$ hiperventilación moderada y $<30 \mathrm{mmHg}$ hiperventilación severa. ${ }^{34}$

Otras herramientas apropiadas para todos los subtipos de la respiración disfuncional son:

Método de evaluación manual del movimiento respiratorio: evalúa extensión y equilibrio de la dirección de movimiento vertical a lateral percibida por las manos del médico en la parte posterior y lateral de la caja torácica inferior. ${ }^{35}$

Prueba de contención del aliento: se mide el tiempo de contención del aliento después de una exhalación normal en capacidad residual funcional. A esto se le conoce como control de pausa. El tiempo de contención del alien-

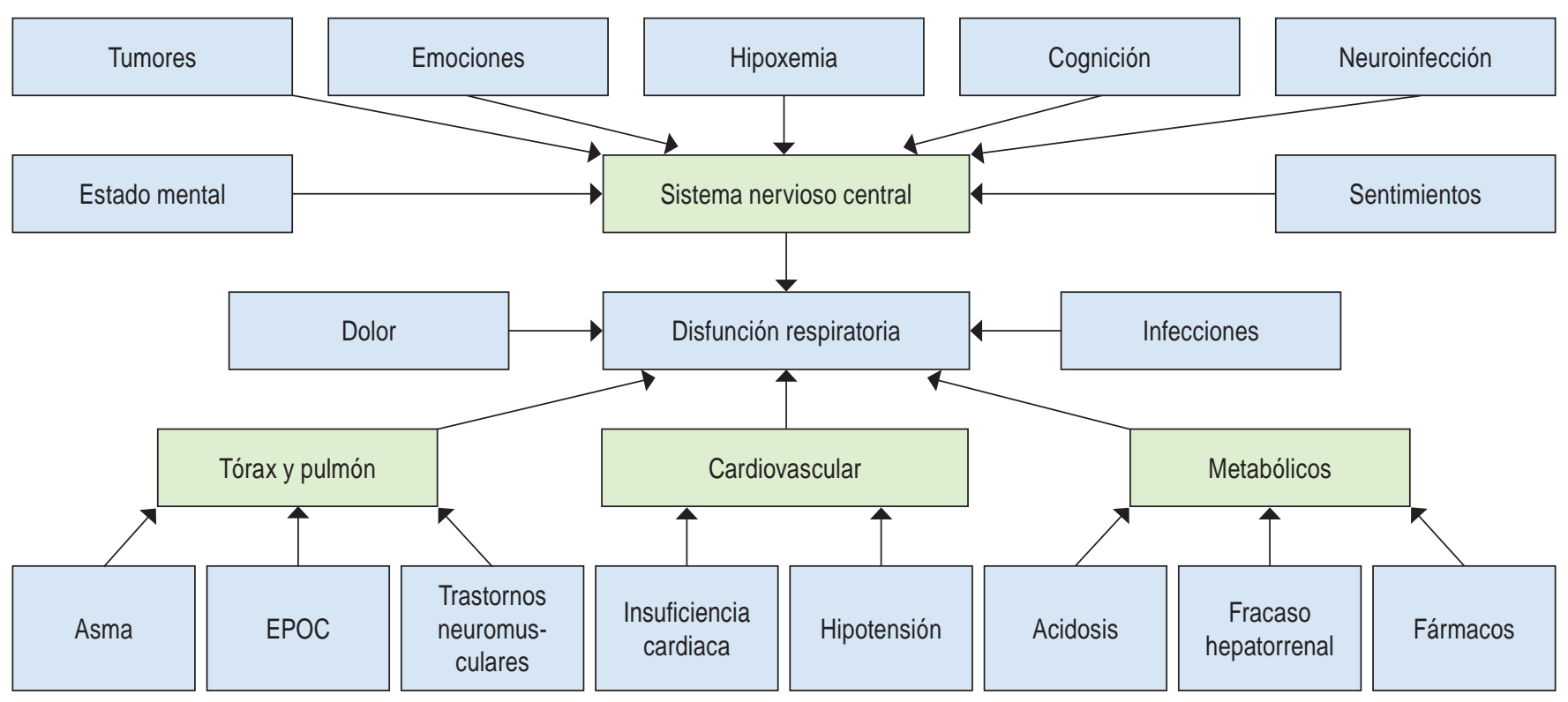

Figura 3: Factores precipitantes de la disfunción respiratoria. ${ }^{12,13,24}$ $\mathrm{EPOC}$ = enfermedad pulmonar obstructiva crónica. 
Tabla 1: Tratamientos dirigidos hacia la respiración disfuncional en paciente con EPOC.

\begin{tabular}{|c|c|c|}
\hline $\begin{array}{l}\text { Tipo de ejercicio } \\
\text { respiratorio }\end{array}$ & Efecto sobre la mecánica de la respiración & Beneficios \\
\hline $\begin{array}{l}\text { Espiración lenta y } \\
\text { profunda }\end{array}$ & $\begin{array}{l}\text { La ventilación alveolar mejora a una frecuencia respiratoria menor y un volumen } \\
\text { tidal mayor. Acorta el tiempo inspiratorio }{ }^{45}\end{array}$ & $\begin{array}{l}\text { Mejora la eficiencia respiratoria y la } \\
\text { saturación de oxígeno en reposo }{ }^{45}\end{array}$ \\
\hline $\begin{array}{l}\text { Respiración con los } \\
\text { labios fruncidos }\end{array}$ & $\begin{array}{l}\text { Aumenta el volumen espirado a comparación de la respiración tidal }{ }^{59} \text { con lo que, } \\
\text { teóricamente, ayudaría a disminuir la hiperinsuflación pulmonar. Comparado } \\
\text { con la respiración espontánea, la espiración con labios fruncidos reduce la ten- } \\
\text { sión de } \mathrm{CO}_{2} \text {, y mejora el volumen tidal y la saturación de oxígeno en reposo }\end{array}$ & $\begin{array}{l}\text { Reduce la disnea en grados variables y } \\
\text { mejora la resistencia física }{ }^{59,60,63}\end{array}$ \\
\hline $\begin{array}{l}\text { Terapia de } \\
\text { relajación }\end{array}$ & $\begin{array}{l}\text { Nace de la observación de que la hiperinflación en la obstrucción de la vía } \\
\text { aérea está dada por una actividad incrementada de los músculos inspiratorios } \\
\text { durante la espiración. }{ }^{65} \\
\text { Teóricamente podría reducir la activación de estos músculos }\end{array}$ & $\begin{array}{l}\text { Mejora en variables como disminución } \\
\text { en la frecuencia cardíaca, respiratoria, } \\
\text { ansiedad y de manera variable en los } \\
\text { grados de disnea }{ }^{65}\end{array}$ \\
\hline $\begin{array}{l}\text { Terapia de músculos } \\
\text { inspiratorios }\end{array}$ & $\begin{array}{l}\text { Incrementa la fuerza y resistencia de los músculos inspiratorios, reduce la } \\
\text { hiperinflación dinámica }{ }^{67}\end{array}$ & $\begin{array}{l}\text { Reduce la disnea y fatiga. }{ }^{67} \text { Aumenta la } \\
\text { tolerancia al ejercicio, mejora el desem- } \\
\text { peño en las actividades de la vida diaria } \\
\text { y una mejoría en la calidad de vida }{ }^{32,69,70}\end{array}$ \\
\hline $\begin{array}{l}\text { Respiración } \\
\text { diafragmática }\end{array}$ & $\begin{array}{l}\text { Mejora el movimiento de la pared torácica y la distribución de la ventilación, } \\
\text { con esto disminuye el costo energético de la respiración, la contribución de los } \\
\text { músculos de las costillas }{ }^{45}\end{array}$ & $\begin{array}{l}\text { Disminuye la disnea y mejora el rendi- } \\
\text { miento en el ejercicio }{ }^{45}\end{array}$ \\
\hline
\end{tabular}

$\mathrm{EPOC}=$ enfermedad pulmonar obstructiva crónica.

to es un indicador de la respuesta ventilatoria a diversos factores bioquímicos, biomecánicos y psicológicos, 31,36,37 un tiempo acortado puede indicar anormalidades en el control respiratorio que resultan en una disfunción respiratoria. ${ }^{38,39}$

Pletismografía respiratoria de inducción: mide los volúmenes respiratorios, tiempos del patrón respiratorio, así como movimiento del tórax y abdomen sin necesidad de una pieza colocada en la boca o un aparato respiratorio. De manera reciente ha sido muy utilizado un sistema portátil que incorpora bandas en un chaleco elastizado, lo que permite un seguimiento ambulatorio continuo con una mínima interferencia en las actividades diarias. ${ }^{35}$ En la actualidad, esta prueba se puede considerar la de referencia para la evaluación del patrón respiratorio. ${ }^{35}$

Pletismografía optoelectrónica (POE): es una técnica de captura de movimiento en 3D que mide el movimiento de la pared torácica de manera no invasiva. Permite la compartimentalización del torso proporcionando significativamente más información sobre el patrón de respiración en comparación con técnicas tradicionales como el análisis de respiración por observación. Es ventajosa sobre otros sistemas de medición similares como pletismografía estruc- turada por luz (PSE), ya que proporciona más información sobre la mecánica de la respiración y se puede utilizar durante el ejercicio, haciéndolo más adecuado para medir el patrón de respiración de los atletas. ${ }^{40}$

Pletismografía estructurada con luz: es una herramienta no invasiva que usa rayos de luz para medir los movimientos del tórax y abdomen. ${ }^{41}$ La luz que proyecta al paciente es una luz en forma de tablero de ajedrez en donde las intersecciones se captan con una cámara a 30 marcos por segundo para hacer una reconstrucción y grabación del movimiento de la pared torácica. Basado en tiempo y proporción de parámetros numéricos, incluido el tiempo inspiratorio, el tiempo espiratorio, tiempo total de respiración y sus relaciones: tiempo inspiratorio/tiempo espiratorio y tiempo inspiratorio/tiempo respiratorio total. También puede medir la asincronía toracoabdominal relativa, asincronía de derecha a izquierda, hemitorácica o contribuciones regionales seleccionadas. ${ }^{41}$ Esta técnica requiere que el paciente esté en reposo. ${ }^{41}$

El adecuado empleo de estas herramientas de diagnóstico en combinación pueden acercar hacia el mecanismo alterado en el patrón respiratorio del paciente, lo que resultaría en una selección adecuada del tratamiento. 


\section{Disfunción en la respiración no asociada con enfermedad orgánica}

La asociación entre este trastorno y la psicopatología es clara. Se han descrito asociaciones con el trastorno de ansiedad, trastorno de pánico, incluso depresión y fobias específicas. ${ }^{42}$ También se ha asociado a rasgos de inferioridad y perfeccionismo. ${ }^{26,42}$

Los factores que hacen que permanezca la hiperventilación son la mala atribución y el pánico, los suspiros como parte de un trastorno de hábito y alguna forma de reinicio fisiológico. ${ }^{26}$

\section{Disfunción en la respiración asociada con enfermedad orgánica}

Algunas enfermedades respiratorias crónicas, como el asma y EPOC, han sido comúnmente asociadas a los patrones de respiración disfuncional.

Asma. El asma tiene un enlace muy cercano con la respiración disfuncional; ha sido relacionada al síndrome de hiperventilación principalmente, ${ }^{6,26}$ pero también a la respiración torácica dominante. ${ }^{43}$

EPOC. Se conoce que estos pacientes presentan variadas alteraciones en el patrón respiratorio, entre las cuales se encuentran: una respiración toracodominante, ${ }^{30}$ respiración superficial y rápida, ${ }^{42}$ respiración abdominal forzada ${ }^{44}$ y asincronía toracoabdominal. ${ }^{45}$

Aunque varias enfermedades orgánicas (ej., enfermedades pulmonares restrictivas) pueden estar asociadas con la respiración disfuncional, éstas han sido escasamente estudiadas.

\section{Tratamiento}

El manejo en general consiste en el reentrenamiento de la respiración, técnicas de relajación y educación acerca de la respiración disfuncional. ${ }^{46}$

Puede agregarse el apoyo psicológico o psiquiátrico según sea el caso. ${ }^{26}$

\section{Fisioterapia respiratoria}

Las técnicas que han sido descritas son:

La técnica de Buteyko: se ha usado para tratar el síndrome de hiperventilación principalmente, pero también se diseñó para varios síntomas y trastornos entre los cuales se encuentra la hipertensión, diabetes, apnea del sueño, ansiedad, depresión, insomnio y epilepsia. ${ }^{47}$ Involucra una combinación de respiraciones lentas y reducidas en volumen, además de ejercicios que involucran sostener la respiración para normalizar el patrón respiratorio y reducir la hiperventilación con el tiempo. ${ }^{30,48-50}$ Se ha visto que reduce los síntomas y el uso de broncodilatador en pacientes con asma. ${ }^{51}$ Más información sobre este método está disponible en https://correctbreathing.com/buteyko-method/

El método de Papworth: consiste en una respiración diafragmática con un énfasis en la respiración nasal lenta y controlada. Este método se enfoca en pacientes con hiperventilación e hiperinflación. ${ }^{52}$

\section{Disfunción respiratoria primaria}

En estas situaciones clínicas, el médico debe notar la relación de los síntomas de respiración disfuncional con la patología psiquiátrica y abordar el caso junto con psicología si es necesario.

La respiración lenta, profunda y controlada parece ser adecuada para muchos casos de respiración disfuncional primaria. Esta causa una inhibición del sistema nervioso simpático y provoca niveles más altos de sincronización cardiorrespiratoria, lo cual puede influir los estados emocionales $^{53}$ y resultar en un beneficio para el paciente.

Otras terapias respiratorias que podrían ser útiles debido al enlace que tienen con la patología psiquiátrica son: las terapias Pranayama (técnica de respiración empleada en yoga) y la respiración Sudarshan Kriya Yogic (SKY). Éstas han demostrado reducciones en el estrés, ansiedad y/o síntomas depresivos en varios estudios. ${ }^{53-56}$

\section{Disfunción respiratoria secundaria}

Asma. En general, una tendencia positiva a la mejora con los ejercicios respiratorios se muestra en la literatura, y en la mayoría la función pulmonar se ve mejorada sólo de manera escasa, sin embargo, existe una mejora significativa en los cuestionarios de disfunción respiratoria y calidad de vida. Una revisión sistemática comparó en pacientes con asma los siguientes tratamientos: yoga, el reentrenamiento de la respiración, el método de Papworth, el método Buteyko, Pranayama y la respiración diafragmática profunda; ésta mostró una mejora en la calidad de vida a los 4-6 meses y una reducción en los síntomas de hiperventilación. ${ }^{57}$

Otra revisión sistemática que estudió el efecto de la técnica de Buteyko encontró una disminución significativa en el uso de los beta 2 agonistas, disminución en el uso de corticosteroides inhalados y una mejora en la calidad de vida. El entrenamiento de los músculos respiratorios encontró una reducción significativa en el uso de medicamentos broncodilatadores. También demostró el beneficio del yoga, la técnica de respiración Buteyko y el entrenamiento respiratorio con fisioterapia para mejorar la calidad de vida relacionada con el asma..$^{58}$

EPOC. Se ha descrito una gran diversidad de ejercicios respiratorios usados en EPOC entre los que se encuentran: espiración activa, espiración lenta y profunda, respiración 
con los labios fruncidos, terapia de relajación, respiración con inclinación hacia adelante, inspiratoria y entrenamiento de los músculos espiratorios y respiración diafragmática (Tabla 1).

Dentro de las técnicas que cuentan con mayor evidencia está la respiración con los labios fruncidos. Ésta consiste en una espiración lenta y controlada a través de la boca con los labios medio abiertos, ${ }^{25}$ por lo que se alarga el tiempo espiratorio, permitiendo teóricamente una disminución en la retención de $\mathrm{CO}_{2}$ y consiguiente mejora en la oxigenación. Esto se ha demostrado clínicamente con estudios que emplearon la prueba de la caminata, en los que se vio una mejor oxigenación, mayor cantidad recorrida ${ }^{59}$ y una reducción en la percepción de la disnea durante la prueba. ${ }^{60}$

Los descritos de manera directa o indirecta ayudarían a mejorar la respiración disfuncional. Para estos pacientes el conocimiento del patrón respiratorio al igual que otras variables ayudan a adecuar y realizar programas de ejercicios respiratorios para cada paciente. Este conocimiento aunado al patrón respiratorio específico obtenido con las nuevas pruebas específicas nos permitirán detallar la terapéutica.

\section{Otras asociaciones}

Existe información acerca de la asociación de la respiración disfuncional y el dolor de espalda. Debido a que estos pacientes, como se mencionó anteriormente, pueden tener tensión muscular por la alteración en el patrón respiratorio, se han empezado a implementar técnicas de masaje como coadyuvante en el tratamiento. ${ }^{61}$

Un estudio por Jones et al. ${ }^{62}$ comparó dos grupos de pacientes con disfunción respiratoria; a un grupo se le aplicaron solamente ejercicios de reentrenamiento de la respiración y al otro grupo con terapia manual aunada a los ejercicios. Ambos grupos tuvieron mejoras considerables en cuanto a puntajes en el cuestionario de Nijmegen, disminución en puntajes de cuestionarios de ansiedad, mejora en resultados de caminata de seis minutos, prueba de contención de la respiración y algunas mediciones esqueléticas. Sin embargo, este estudio no mostró un beneficio adicional cuando se agregó la terapia manual a los ejercicios.

\section{DISCUSIÓN}

La respiración disfuncional representa un problema importante para la sociedad en la actualidad, con gran carga en la calidad de vida y para el sistema de salud. La interpretación de la evidencia actual es difícil debido a la ausencia de consenso en cuanto a la definición; sin embargo, existe un amplio campo para el desarrollo de estos clínicos que ayuden a la adecuada comprensión del síndrome y permitan la mitigación de las grandes consecuencias de éste. Conside- ramos necesario como primer paso conducir estudios que permitan la definición del síndrome y su organización en distintos tipos, esto nos encaminará a detallar las estrategias de tratamiento que existen actualmente para cada tipo de patrón respiratorio anormal.

Dada la heterogeneidad de la respiración disfuncional y la cercana asociación que existe con la psicopatología, aportará mucho el conocimiento que pueda derivar de estudios cualitativos sobre los factores relacionados y nos proveerán de otras perspectivas del problema. Se advienen grandes descubrimientos para un trastorno que siempre estuvimos viendo, sin embargo, no estábamos prestando atención. Éstos podrán iluminar una nueva perspectiva del estado fisiológico del ser humano, que comprenda más que lo que actualmente se ha tomado en cuenta y ayudaría a que la sociedad y el médico enfaticen sobre la importancia del cuidado del patrón respiratorio.

\section{REFERENCIAS}

1. Barker N, Everard ML. Getting to grips with "dysfunctional breathing". Paediatr Respir Rev. 2015;16(1):53-61. doi: 10.1016/j.prrv.2014.10.001.

2. Boulding R, Stacey R, Niven R, Fowler SJ. Dysfunctional breathing: a review of the literature and proposal for classification. Eur Respir Rev. 2016;25(141):287-294. doi: 10.1183/16000617.0088-2015.

3. Han JN, Stegen K, De Valck C, Clément J, Van de Woestijne KP. Influence of breathing therapy on complaints, anxiety and breathing pattern in patients with hyperventilation syndrome and anxiety disorders. J Psychosom Res. 1996;41(5):481-493. doi: 10.1016/ S0022-3999(96)00220-6.

4. Gilbert C. Emotional sources of dysfunctional breathing. J Bodyw Mov Ther. 1998;2(4):224-230. doi: 10.1016/S1360-8592(98)80019-3.

5. Thomas M, McKinley RK, Freeman E, Foy C, Price D. The prevalence of dysfunctional breathing in adults in the community with and without asthma. Prim Care Respir J. 2005;14(2):78-82. doi: 10.1016/j. pcrj.2004.10.007.

6. DeGuire S, Gevirtz R, Hawkinson D, Dixon K. Breathing retraining: a three-year follow-up study of treatment for hyperventilation syndrome and associated functional cardiac symptoms. Biofeedback Self Regul. 1996;21(2):191-198. doi: 10.1007/BF02284695.

7. Lum LC. Hyperventilation: the tip and the iceberg. J Psychosom Res. 1975;19(5-6):375-383. doi: 10.1016/0022-3999(75)90017-3.

8. Brashear RE. Hyperventilation syndrome. Lung. 1983;161(5):257-273. doi: 10.1007/BF02713872.

9. Morgan MD. Dysfunctional breathing in asthma: is it common, identifiable and correctable? Thorax. 2002;57(Suppl 2):II31-II35. doi: 10.1136/thorax.57.suppl_2.ii31.

10. Law N, Ruane LE, Low K, Hamza K, Bardin PG. Dysfunctional breathing is more frequent in chronic obstructive pulmonary disease than in asthma and in health. Respir Physiol Neurobiol. 2018;247:2023. doi: 10.1016/j.resp.2017.08.011.

11. Han JN, Stegen K, Simkens K, Cauberghs M, Schepers R, Van den Bergh $\mathrm{O}$, et al. Unsteadiness of breathing in patients with hyperventilation syndrome and anxiety disorders. Eur Respir J. 1997;10(1):167-176. doi: 10.1183/09031936.97.10010167.

12. Del Negro CA, Funk GD, Feldman JL. Breathing matters. Nat Rev Neurosci. 2018;19(6):351-367. doi: 10.1038/s41583-018-0003-6. 
13. Boiten FA, Frijda NH, Wientjes CJE. Emotions and respiratory patterns: review and critical analysis. Int J Psychophysiol. 1994;17(2):103-128. Available in: https://doi.org/10.1016/0167-8760(94)90027-2

14. Gómez $\mathrm{CMH}$. Clinical intensive care medicine. Introductory series in medicine: Volume 1. Singapore: World Scientific Publishing; 2014. 41-69. Available in: https://doi.org/10.1142/p640

15. Tuck SA, Dort JC, Remmers JE. Braking of expiratory airflow in obese pigs during wakefulness and sleep. Respir Physiol. 2001;128(2):241245. doi: 10.1016/s0034-5687(01)00302-4.

16. Shelledy DC, Peters JI, Proud KC. Mechanical ventilation. Burlington, MA: Jones \& Bartlett Learning; 2020. p. 256, 257. Available in: https:// www.jblearning.com/catalog/productdetails/9781284125931

17. Dutschmann M, Jones SE, Subramanian HH, Stanic D, Bautista TG. The physiological significance of post inspiration in respiratory control. Prog. Brain Res. 2014;212:113-130. doi: 10.1016/B978-0-444-634887.00007-0.

18. Sapienza CM, Lindsey BG, Morris KF, Davenport PW, Bolser DC. Coordination of cough and swallow: a meta-behavioral response to aspiration. Respir Physiol Neurobiol. 2013;189(3):543-51. doi: 10.1016/j.resp.2013.08.009.

19. Shannon R, Baekey DM, Morris KF, Nuding SC, Segers LS, Lindsey BG. Production of reflex cough by brainstem respiratory networks. Pulm Pharmacol Ther. 2004;17(6):369-376. doi: 10.1016/j. pupt.2004.09.022.

20. Bautista TG, Sun QJ, Pilowsky PM. The generation of pharyngeal phase of swallow and its coordination with breathing: interaction between the swallow and respiratory central pattern generators. Prog Brain Res. 2014:212:253-275. doi: 10.1016/B978-0-444-63488-7.00013-6.

21. Wheeler Hegland K, Huber JE, Pitts T, Davenport PW, Sapienza $\mathrm{CM}$. Lung volume measured during sequential swallowing in healthy young adults. J Speech Lang Hear Res. 2011;54(3):777-786. doi: 10.1044/1092-4388(2010/09-0237).

22. Jean A. Brain stem control of swallowing: neuronal network and cellular mechanisms. Physiol Rev. 2001;81(2):929-969. doi: 10.1152/ physrev.2001.81.2.929.

23. Pitts T, Bolser D, Rosenbek J, Troche M, Okun MS, Sapienza CM. Impact of expiratory muscle strength training on voluntary cough and swallow function in Parkinson disease. Chest. 2009;135(5):1301-1308 doi: 10.1378/chest.08-1389.

24. Hernandez-Miranda LR, Ruffault PL, Bouvier JC, Murray AJ, MorinSurun MP, Zampieri N, et al. Genetic identification of a hindbrain nucleus essential for innate vocalization. Proc Natl Acad Sci USA. 2017;114(30):8095-8100. doi: 10.1073/pnas.1702893114.

25. Perpiñá-Tordera M. Síndrome de hiperventilación y asma. Arch Bronconeumol. 2004;40(Supl 3):6-10.

26. Gardner WN. The pathophysiology of hyperventilation disorders. Chest. 1996;109(2):516-534. doi: 10.1378/chest.109.2.516.

27. Van Dixhoorn J, Duivenvoorden HJ. Efficacy of Nijmegen Questionnaire in recognition of the hyperventilation syndrome. J Psychosom Res. 1985;29(2):199-206. doi: 10.1016/0022-3999(85)90042-x.

28. Vidotto LS, Carvalho CRF, Harvey A, Jones M. Dysfunctional breathing: what do we know? J Bras Pneumol. 2019;45(1):e20170347. doi: 10.1590/1806-3713/e20170347.

29. van Dixhoorn J, Folgering H. The Nijmegen Questionnaire and dysfunctional breathing. ERJ Open Res. 2015;1(1):00001-2015 doi: 10.1183/23120541.00001-2015.

30. Courtney R, van Dixhoorn J, Greenwood KM, Anthonissen EL. Medically unexplained dyspnea: partly moderated by dysfunctional (thoracic dominant) breathing pattern. J Asthma. 2011;48(3):259-265 doi: 10.3109/02770903.2011.554942.

31. Han JN, Schepers R, Stegen K, Van den Bergh O, Van de Woestijne KP. Psychosomatic symptoms and breathing pattern. J Psychosom Res. 2000;49(5):319-333. doi: 10.1016/s0022-3999(00)00165-3.

32. Hornsveld HK, Garssen B, Dop MJ, van Spiegel PI, de Haes JC. Double-blind placebo-controlled study of the hyperventilation provocation test and the validity of the hyperventilation syndrome. Lancet. 1996;348(9021):154-158. doi: 10.1016/s01406736(96)02024-7.

33. Hornsveld H, Garssen B. The low specificity of the hyperventilation provocation test. J Psychosom Res. 1996;41(5):435-449. doi: 10.1016/ s0022-3999(96)00195-x.

34. Delapille P, Verin E, Tourny-Chollet C, Pasquis P. Breath-holding time: effects of non-chemical factors in divers and non-divers. Pflugers Arch. 2001;442(4):588-594. doi: 10.1007/s004240100568.

35. Gardner WN, Meah MS, Bass C. Controlled study of respiratory responses during prolonged measurement in patients with chronic hyperventilation. Lancet. 1986;328(8511):826-830. Available in: https://doi.org/10.1016/S0140-6736(86)92867-9

36. Courtney R, van Dixhoorn J, Cohen M. Evaluation of breathing pattern: comparison of a manual assessment of respiratory motion (MARM) and respiratory induction plethysmography. Appl Psychophysiol Biofeedback. 2008;33(2):91-100. doi: 10.1378/chest.84.3.286.

37. Tobin MJ, Chadha TS, Jenouri G, Birch SJ, Gazeroglu HB, Sackner MA. Breathing patterns. Chest. 1983;84(3):286-294. doi: 10.1378/ chest.84.3.286

38. Verschakelen JA, Demedts MG. Normal thoracoabdominal motions. Influence of sex, age, posture, and breath size. Am J Respir Crit Care Med. 1995;151(2 Pt 1):399-405. doi: 10.1164/ajrccm.151.2.7842198.

39. Lin YC, Lally DA, Moore TO, Hong SK. Physiological and conventional breath-hold breaking points. J Appl Physiol. 1974;37(3):291-296. doi: 10.1152/jappl.1974.37.3.291.

40. Lumb A. Control of breathing. In: Lumb AB, ed. Nunn's respiratory physiology. 8th edition. Oxford: Butterworth-Heinemann; 2017: 51-72.

41. Lauhkonen E, Cooper BG, lles R. Mini review shows that structured light plethysmography provides a non-contact method for evaluating breathing patterns in children. Acta Paediatr. 2019;108(8):1398-1405. doi: 10.1111/apa.14769.

42. Hagman C, Janson C, Emtner M. A comparison between patients with dysfunctional breathing and patients with asthma. Clin Respir J. 2008;2(2):86-91. doi: 10.1111/j.1752-699X.2007.00036.x.

43. Lougheed MD, Fisher T, O'Donnell DE. Dynamic hyperinflation during bronchoconstriction in asthma: implications for symptom perception. Chest. 2006;130(4):1072-1081. doi: 10.1378/chest.130.4.1072.

44. Bott J, Blumenthal S, Buxton M, Ellum S, Falconer C, Garrod R, et al. Guidelines for the physiotherapy management of the adult, medical, spontaneously breathing patient. Thorax. 2009;64(Suppl 1):i1-i52. doi: $10.1136 /$ thx.2008.110726.

45. Gosselink R. Breathing techniques in patients with chronic obstructive pulmonary disease (COPD). Chron Respir Dis. 2004;1(3):163-172. doi: 10.1191/1479972304cd020rs.

46. Ferrari K, Goti P, Duranti R, landelli I, Misuri G, Mancini M, et al. Breathlessness and control of breathing in patients with COPD. Monaldi Arch Chest Dis. 1997;52(1):18-23.

47. Cluff RA. Chronic hyperventilation and its treatment by physiotherapy: discussion paper. J R Soc Med. 1984;77(10):855-862. doi: 10.1177/014107688407701011. 
48. Stark J, Stark R. The carbon dioxide syndrome. Coorparoo, Australia: Buteyko On Line Ltd.; 2002;36(2):59-63.

49. Bruton A, Lewith GT. The Buteyko breathing technique for asthma: a review. Complement Ther Med. 2005;13(1):41-46. Available in: https:// doi.org/10.7322/jhgd.v30.10381

50. Cooper S, Oborne J, Newton S, Harrison V, Thompson Coon J, Lewis $\mathrm{S}$, et al. Effect of two breathing exercises (Buteyko and pranayama) in asthma: a randomised controlled trial. Thorax. 2003;58(8):674-679. doi: 10.1136/thorax.58.8.674.

51. Courtney R. Strengths, weaknesses, and possibilities of the Buteyko breathing method. Biofeedback. 2008:36(2):59-63.

52. Holloway EA, West RJ. Integrated breathing and relaxation training (the Papworth method) for adults with asthma in primary care: a randomised controlled trial. Thorax. 2007;62(12):1039-1042. doi: 10.1136/thx.2006.076430

53. Jerath R, Crawford MW, Barnes VA, Harden K. Self-regulation of breathing as a primary treatment for anxiety. Appl Psychophysiol Biofeedback. 2015;40(2):107-115. doi: 10.1007/s10484-015-9279-8.

54. Bhimani NT, Kulkarni NB, Kowale A, Salvi S. Effect of Pranayama on stress and cardiovascular autonomic function. Indian J Physiol Pharmacol. 2011;55(4):370-377.

55. Brown RP, Gerbarg PL. Sudarshan Kriya Yogic breathing in the treatment of stress, anxiety, and depression: Part II-clinical applications and guidelines. J Altern Complement Med. 2015;11(4):711717. doi: 10.1089/acm.2005.11.711.

56. Marshall RS, Basilakos A, Williams T, Love-Myers K. Exploring the benefits of unilateral nostril breathing practice post-stroke: attention, language, spatial abilities, depression, and anxiety. J Altern Complement Med. 2014;20(3):185-194. doi: 10.1089/acm.2013.0019.

57. Santino TA, Chaves GS, Freitas DA, Fregonezi GA, Mendonca KM. Breathing exercises for adults with asthma. Cochrane Database Syst Rev. 2020;3(3):CD001277. doi: 10.1002/14651858.CD001277.pub4.

58. Burgess J, Ekanayake B, Lowe A, Dunt D, Thien F, Dharmage SC. Systematic review of the effectiveness of breathing retraining in asthma management. Expert Rev Respir Med. 2011 5(6):789-807. doi: 10.1586/ers.11.69.

59. Faager G, Stahle A, Larsen FF. Influence of spontaneous pursed lips breathing on walking endurance and oxygen saturation in patients with moderate to severe chronic obstructive pulmonary disease. Clin Rehabil. 2008;22(8):675-683. doi: 10.1177/0269215508088986.
60. Nield MA, Soo Hoo GW, Roper JM, Santiago S. Efficacy of pursedlips breathing: a breathing pattern retraining strategy for dyspnea reduction. J Cardiopulm Rehabil Prev. 2007;27(4):237-244. doi: 10.1097/01.HCR.0000281770.82652.cb.

61. Hondras MA, Linde K, Jones AP. Manual therapy for asthma. Cochrane Database Syst Rev. 2005;18(2). doi: 10.1002/14651858. CD001002.pub2.

62. Jones M, Troup F, Nugus J, Roughton M, Hodson M, Rayner C, et al. Does manual therapy provide additional benefit to breathing retraining in the management of dysfunctional breathing? A randomised controlled trial. Disabil Rehabil. 2015;37(9):763-770. doi: 10.3109/09638288.2014.941020.

63. Breslin EH. The pattern of respiratory muscle recruitment during pursed-lip breathing. Chest. 1992;101(1):75-78. doi: 10.1378/ chest.101.1.75.

64. Tiep BL, Burns M, Kao D, Madison R, Herrera J. Pursed lips breathing training using ear oximetry. Chest. 1986;90(2):218-221. doi: 10.1378/ chest.90.2.218.

65. Gift AG, Moore T, Soeken K. Relaxation to reduce dyspnea and anxiety in COPD patients. Nurs Res. 1992;41(4):242-246.

66. Barach AL. Chronic obstructive lung disease: postural relief of dyspnea. Arch Phys Med Rehabil. 1974;55(11):494-504.

67. Petrovic M, Reiter M, Zipko H, Pohl W, Wanke T. Effects of inspiratory muscle training on dynamic hyperinflation in patients with COPD. Int J Chron Obstruct Pulmon Dis. 2012;7:797-805. doi: 10.2147/COPD. S23784.

68. Sharp JT, Drutz WS, Moisan T, Foster J, Machnach W. Postural relief of dyspnea in severe chronic obstructive pulmonary disease. Am Rev Respir Dis. 1980;122(2):201-211. doi: 10.1164/arrd.1980.122.2.201.

69. Geddes EL, O'Brien K, Reid WD, Brooks D, Crowe J. Inspiratory muscle training in adults with chronic obstructive pulmonary disease: an update of a systematic review. Respir Med. 2008;102(12):17151729. doi: 10.1016/j.rmed.2008.07.005.

70. Shoemaker MJ, Donker S, Lapoe A. Inspiratory muscle training in patients with chronic obstructive pulmonary disease: the state of the evidence. Cardiopulm Phys Ther J. 2009;20(3):5-15.

Conflicto de intereses: Los autores declaran no tener conflicto de intereses. 